\title{
Gênero Hippopsis (Coleoptera, Cerambycidae, Lamiinae): chave para as espécies, sinonímia e descrição de espécies novas
}

\author{
Ubirajara R. Martins ${ }^{1,3}$ \& Maria Helena M. Galileo ${ }^{2,3}$
}

\begin{abstract}
${ }^{1}$ Museu de Zoologia, Universidade de São Paulo. Caixa Postal 42494, 04218-970 São Paulo-SP, Brasil.
${ }^{2}$ Museu de Ciências Naturais, Fundação Zoobotânica do Rio Grande do Sul. Caixa Postal 1188, 90001-970 Porto Alegre-RS, Brasil. ${ }^{3}$ Pesquisador do CNPq.
\end{abstract}

\begin{abstract}
Genus Hippopsis (Coleoptera, Cerambycidae, Lamiinae): key to species, synonym, and description of new species. New species described: $H$. iuasanga from Ecuador and $H$. araujoi from Brasil (Bahia). A key to species is given, and H. lineolatus Lepeletier \& Audinet-Serville, 1825, H. lemniscata boliviana Breuning, 1962, H. insularis Breuning, 1962, are not included, pending on examination of holotypes; species not studied and included by literature: $H$. septemvittata Breuning, 1940 and H. albicans Breuning, 1940. Hippopsis solangeae Carvalho, 1981 is considered a synonym of $H$. prona Bates, 1866 and $H$. insularis Breuning, 1962 is revalidated. Thirty six species are illustrated.
\end{abstract}

KEYWORDS. Hippopsini; key; synonym; taxonomy.

RESUMO. Gênero Hippopsis (Coleoptera, Cerambycidae, Lamiinae): chave para as espécies, sinonímia e descrição de espécies novas. São descritas: $H$. iuasanga do Equador e $H$. araujoi do Brasil (Bahia). Acrescenta-se uma chave para espécies na qual não foram incluídas as espécies só identificáveis mediante o exame dos holótipos: H. lineolatus Lepeletier \& Audinet-Serville, 1825; H. lemniscata boliviana Breuning, 1962; H. insularis Breuning, 1962. Espécies não examinadas que são incluídas com base na literatura: H. septemvittata Breuning, 1940 e H. albicans Breuning, 1940. Hippopsis solangeae Carvalho, 1981 é considerada sinônimo de H. prona Bates, 1866 e H. insularis Breuning, 1962, é revalidada. Trinta e seis espécies são ilustradas.

PALAVRAS-CHAVE. Chave; Hippopsini; sinonímia; taxonomia.

O tratamento apropriado das 38 espécies do gênero Hippopsis Lepeletier \& Audinet-Serville, 1825, envolve problemas. O maior deles é que a maioria das espécies descritas, redescritas ou comentadas por Breuning (1962) é de reconhecimento difícil ou impossível. Uma revisão do gênero, com base no exame dos holótipos seria muito desejável, mas como não tivemos acesso a eles, essa tarefa torna-se muito difícil. Para ter-se idéia de como a revisão de Breuning (op. cit.) é superficial, basta dizer que ele não observou e não tratou da pilosidade diferenciada ou dos orifícios que aparecem no urosternito I de diversas espécies.

Breuning (1962) também propôs a subdivisão de Hippopsis em dois subgêneros: Hippopsis s. str. e Megacera AudinetServille, 1835, mas adotamos a classificação anterior (Aurivillius, 1923), onde ambas as entidades são consideradas gêneros.

Apresentamos uma chave preliminar para as espécies de Hippopsis na qual deixamos de introduzir $H$. lineolatus Lepeletier \& Audinet-Serville, 1825, H. lemniscata boliviana Breuning, 1962 e H. insularis porque não temos idéia do que possam ser. Hippopsis septemvittata Breuning, 1940 e $H$. albicans Breuning, 1940 foram tentativamente introduzidas com base na literatura.

Além da chave para as espécies, descrevemos duas, sinonimizamos uma e apresentamos figuras de todas as espécies tratadas.

O material estudado pertence ao Museu de Zoologia, Universidade de São Paulo, São Paulo (MZSP) e ao Museu de
Ciências Naturais, Fundação Zoobotânica do Rio Grande do Sul, Porto Alegre (MCNZ). Entretanto, siglas de outras instituições são mencionadas no texto e correspondem: BMNH, The Natural History Museum, Londres; FTHC, Coleção F. T. Hovore, Santa Clarita; MNHN, Muséum National d'Histoire Naturelle, Paris; NHRS, Naturhistoriska Riksmuseet, Estocolmo; ZSMC, Zoologische Staatssammlung des Bayerischen Staates, München.

\section{HISTÓRICO}

A primeira espécie descrita em Hippopsis foi no gênero Saperda Fabricius, 1775: Saperda lemniscata Fabricius, 1801, procedente da "Carolina", Estados Unidos. O gênero foi descrito por Lepeletier \& Audinet-Serville (1825) com base em $H$. lineolatus do Brasil, portanto a espécie-tipo do gênero por monotipia, mas com uma observação que $H$. lineolatus poderia ser a espécie de Fabricius.

Guérin-Méneville (1844) descreveu, também do Brasil, $H$. pradieri.

Bates (1866) descreveu $H$. apicalis (originalmente em Megacera Audinet-Serville, 1835), H. fractilinea, H. griseola, $H$. prona e H. truncatella todas da Amazônia brasileira. Observou que as espécies de Hippopsis "are parasitic on the slender branches of trees. They choose, however, the most slender twigs, and cling them so closely by their short stout legs and elongate claws as to be difficult of detection." Anotamos que Bates (1866) não chegou a observar o notório 
orifício que $H$. prona tem no urosternito I.

Berg (1889) acrescentou H. monachica procedente de Buenos Aires, Argentina e Aurivillius (1900) adicionou $H$. meinerti de Caracas, Venezuela e, em 1920, H. quinquelineata do Brasil meridional.

Breuning (1940) descreveu sumariamente oito espécies: H. albicans (Santa Catarina, Blumenau), espécie que jamais foi citada e nunca mais foi coletada da qual só se conhece o holótipo (ZSMC); H. assimilis da Venezuela; H. densepunctata da Bahia (Santo Antonio da Barra, atual Condeúba); $H$. femoralis de São Paulo (Alto da Serra); H. macrophthalma do Amazonas (Tefé); H. quadrivittata e H. septemlineata ambas da Colômbia e H. septemvittata, do Brasil ( sem outras especificações).

Em 1955, Breuning acrescentou mais uma espécie: $H$. freyi de Trinidad. Esta espécie foi considerada na sinonímia de $H$. meinerti por Galileo \& Martins (1988a: 185), mas revalidada por Martins \& Galileo (2003).

Breuning (1962) procedeu a uma revisão do gênero quando apresentou a "Révision des Agapanthiini Muls. Américains". Propôs a subdivisão de Hippopsis em dois subgêneros: Hippopsis s. str. e Megacera Audinet-Serville, 1835. Não nos parece que esses subgêneros sejam viáveis e adotamos a classificação anterior (Aurivillius, 1923), onde ambas as entidades são consideradas gêneros, que se distinguem principalmente pelo comprimento do protórax, mais longo do que largo em Hippopsis e tão longo quanto largo em Megacera. Breuning (1962) também apresentou uma chave para as espécies de Hippopsis com equívocos: omitiu $H$. monachica Berg, 1899, apesar da mesma constar do catálogo de Aurivillius (1923); utilizou caracteres de difícil interpretação: espículo na lado interno das extremidades elitrais (varia e pode desaparecer); élitros densa e finamente pontuados ou élitros muito densa e muito finamente pontuados; comprimento dos lobos oculares inferiores com relação ao comprimento das genas e comprimento do antenômero III. Além disso, não observou caráter muito conspícuo, qual seja, as modificações no urosternito I e nos metafêmures dos machos. Também considerou $H$. meinerti como morpho de H. lemniscata e descreveu, sumariamente, três subespécies de H. lemniscata: boliviana (da Bolívia, Mapiri, que figurou, mas é pouco elucidativa), insularis (de Trinidad) e tobagoensis (de Tobago); publicou ainda $H$. gilmouri (de Agua Blanca, Salta, Argentina, mas a figura apresentada também serve para várias espécies).

Carvalho (1981a, 1981b) descreveu duas espécies: $H$. solangeae do Equador, Peru, Bolívia e Brasil (largamente distribuída), Paraguai e Argentina e H. pallida de Córdoba, Argentina.

Galileo \& Martins (1988a, 1988b, 1988c, 1988d, 1988e) dividiram Hippopsis em sete grupos, forneceram chaves para sua identificação e para o reconhecimento de suas espécies. Adotaram caracteres pela primeira vez: nos machos, orifícios e modificação da pubescência no urosternito I e forma dos metafêmures; presença de rugas no pronoto; ápices dos élitros emarginados e biespinhosos. Publicaram, ainda, a descrição de H. tuberculata e H. pertusa (Mata Atlântica), H. tremata
(México a Costa Rica), H. pubiventris [Peru, Brasil (largamente distribuída)], $H$. minima (Maranhão), $H$. fratercula (Pernambuco e Bahia), H. rabida (São Paulo) e H. renodis (Rio de Janeiro). Galileo \& Martins (1998a) sinonimizaram $H$. lemniscata tobagoensis com H. meinerti.

Tavakilian (1987), baseado no estudo do holótipo (NHRS), constatou que $H$. tremata era sinônima de $H$. meinerti.

Em 1994, Martins \& Galileo publicaram a descrição de $H$. mourai (Brasil, Tocantins), em 1995, H. ocularis (Bolívia) e, em 1998, de H. campaneri (Brasil, Goiás). Os mesmos autores (2003) acrescentaram cinco espécies ao gênero: H. bivittata (Peru), H. nigroapicalis (Equador), H. brevicollis (Brasil, Minas Gerais), H. arriagadai (Paraguai) e H. tibialis (Brasil, São Paulo). Além disso, revalidaram $H$. freyi que saiu da sinonímia da H. meinerti.

Monné (2001) arrolou as plantas-hospedeiras de quatro espécies. Hippopsis lemniscata, tem quantidade considerável de plantas das famílias Amaranthaceae, Asteraceae (a com maior número de plantas-hospedeiras), Curcubitaceae, Leguminosae e Pedaliaceae. Larvas de H. macrophthalma broqueiam Solanum melongena; larvas de $H$. pertusa vivem em Bidens subalternus e Conyza sp. (Asteraceae); de H. prona, em Xanthium strumarium (Asteraceae) e de H. tuberculata, as igualmente Asteraceae, Ageratum conyzoides e Erechtites valerianaefolia.

O gênero Hippopsis contava com 38 espécies (Monné, 2005), com apenas duas assinaladas para a América do Norte e América Central, e 36 espécies para a América do Sul e destas conhecem-se as plantas-hospedeiras de apenas quatro (Monné, 2001). Agora sinonimizamos uma e descrevemos duas espécies de Hippopsis que fica com 39 espécies.

\section{Hippopsis lineolatus Lepeletier \& Audinet-Serville, 1825}

Hippopsis lineolatus Lepeletier \& Audinet-Serville, 1825 in Latreille, 1825: 336.

Hippopsis (Hippopsis) lineolata; Monné, 2005: 251 (cat.).

Hippopsis lineatus; Guérin-Méneville, 1844: 246 (erro de grafia).

Depois da descrição original desta espécie do Brasil, três citações apareceram na bibliografia: Erichson (1847) e Kirch (1875) citaram-na para o Peru, sem mais comentários. Aurivillius (1919) registrou-a, também sem comentários, para o Rio Purus, Amazonas.

Aurivillius (1923) sinonimizou H. lineolatus e H. lineatus com H. lemniscata baseado talvez numa suposição de AudinetServille (1825) (vide abaixo). Galileo \& Martins (1988c) revalidaram $H$. lineolatus, porque $H$. lemniscata parece estar restrita aos Estados Unidos, México e Honduras (Monné \& Hovore, 2005) e, certamente, $H$. lineolatus não ocorre nesses países. Galileo \& Martins (1988c) revalidaram H. lineolatus fundamentados na sua procedência. Entretanto, H. lineolatus, cuja descrição original reproduzimos, não pode ser reconhecida.

A descrição original é de Lepeletier \& Audinet-Serville (1825:336):

"Hippopsis fuscus, capitis thoracisque lineis dex elytra 

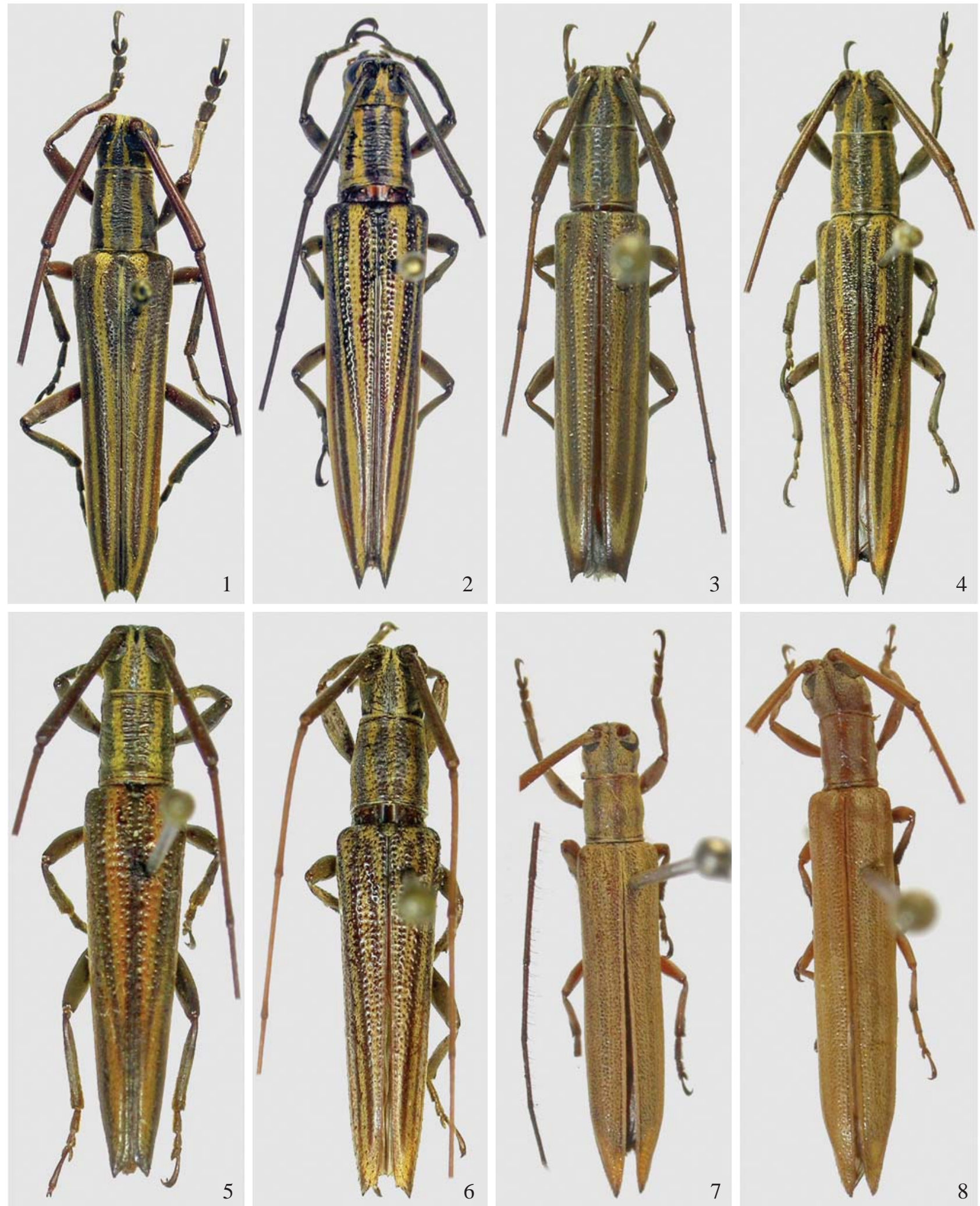

Figs.1-8. 1, Hippopsis assimilis Breuning, 1940, macho, comprimento 22,4 mm; 2, H. fractilinea Bates, 1860, fêmea, 20,7 mm; 3 , H. nigroapicalis Martins \& Galileo, 2003, fêmea, 12,2 mm; 4, H. pradieri Guérin-Méneville, 1844, fêmea, 20,0 mm; 5, H. quadrivittata Breuning, 1940, macho, 13,3 mm; 6, H. septemlineata Breuning, 1940, macho, 13,6 mm; 7, H. monachica Berg, 1889, fêmea, 13,3 mm; 8, H. pallida Carvalho, 1981, parátipo fêmea, 12,4 mm. 
cujusque lineis tribus, interioribus apice conniventibus luteolis.

Longueur 5 lig. Corps d'un brun-noirâtre, ponctué. Antennes ayant plus de deux fois la longueur du corps. Face antérieure de la tête d'un jaune-verdâtre; ses côtes, ceux du corselet et des elytres ayant chacun trois lignes de cette dernière couleur: les intérieures se réunissant à leur extremité. Vers le bout des elytres, qui se terminent en pointe. Côtes de l'abdomen ayant une ligne longitudinale du même jaune que les précédentes. Pattes d'un brun-noirâtre. La couleur jaune de cet insecte est due à des poils courtes et couchés.

Du Brésil.

Note. Cette espèce est peut-être la Saperda lemniscata, Fab. Syst. Eleut. tom. 2, pag. 330, n 69; mais cet auteur dit que son espèce est de la Caroline, et il ne parle pas de la pointe que termine chaque élytre". Audinet-Serville (1835: 42) repetiu essa nota.

Tal descrição serve para quase todas as espécies brasileiras de Hippopsis, portanto, só o exame do holótipo permitirá reconhecê-la. O holótipo encontra-se no BMNH (Monné, 2005: 251).

$H$. lineatus Guérin-Méneville, 1844, sinônima de $H$. lineolatus, é claramente um erro de nome, pois nos comentários após a descrição de $H$. pradieri, Guérin-Méneville faz referência a $H$. lineatus como a espécie de Serville (na realidade, de Lepeletier \& Audinet-Serville).

Se Kirsch, Erichson e Aurivillius estiverem certos, o que é duvidoso já que nenhum deles examinou o holótipo, podemos inferir que $H$. lineolatus é a espécie que ocorre na Amazônia [Peru e Brasil (Amazonas)]; então poderiam eventualmente ser seus sinônimos: $H$. griseola, $H$. macrophthalma ou $H$. prona.

\section{Hippopsis lemniscata boliviana Breuning, 1962}

Hippopsis lemniscata boliviana Breuning, 1962: 10, fig. 1.

Hippopsis (Hippopsis) lemniscata boliviana; Monné, 2005: 251 (cat.).

Esta forma não é subespécie de H. lemniscata. A descrição original é a seguinte:

"ssp. boliviana nov. (Fig. 1).

Comme la forme typique, mais le front seulement légèrement trapéziforme; chaque élytre étiré en un lobe apical sensiblemente plus long. Pronotum avec une étroite bande longitudinale jaune.

Type de Bolivie: Mapiri ao Musée de Stockholm. 1 Paratype de Bolivie (coll. Gilmour)".

A figura apresentada por Breuning (1962: 49, fig. 1) serve apenas para indicar a forma das extremidades dos élitros de per si acuminadas. Não temos idéia do que poderia ser $H$. lemniscata boliviana.

\section{Hippopsis insularis Breuning, 1962 revalidada}

Hippopsis lemniscata insularis Breuning, 1962: 10.

Hippopsis (Hippopsis) lemniscata insularis; Monné, 2005:251 (cat., in syn.)
Monné (2005) considerou esta subespécie na sinonímia de Hippopsis meinerti. Acreditamos que na realidade esta subespécie não pertence a $H$. lemniscata nem a $H$. meinerti. A sua descrição é a seguinte:

"ssp. insularis nov.

Comme la forme typique, mais le scape aussi long que le troisième article des antennes, les lobes inférieurs des yeux deux fois plus longs que le joues, l'angle apical sutural de l'élytre non indiqué.

Long.: $6 \mathrm{~mm}$; larg. $1 \mathrm{~mm}$.

Type de l'Ile Trinidad au British Museum".

\section{Hippopsis septemvittata Breuning, 1940}

Hippopsis septemvittata Breuning, 1940: 81.

Hippopsis (Hippopsis) septemvittata; Monné, 2005: 253 (cat.).

Pela descrição (Breuning, 1940) e redescrição (Breuning, 1962), esta espécie apresenta sete faixas longitudinais de pubescência amarelada no pronoto e élitros arredondados na extremidade, caracteres pouco habituais entre as espécies do gênero. Mas, as anotações de Napp (comunicação pessoal) sobre o holótipo no BMNH dizem o inverso: "protórax sem faixas... metasterno e abdômen normais...". Assim, a inserção da espécie na chave baseia-se na descrição e redescrição, mas não temos certeza se sua colocação está correta.

\section{Hippopsis albicans Breuning, 1940}

Hippopsis albicans Breuning, 1940: 82.

Hippopsis (Hippopsis) albicans; Monné, 2005: 249 (cat.).

Pela redescrição de Breuning (1962), esta espécie tem élitros arredondados no ápice. Este caráter permitiu sua inclusão na chave porque a espécie é desconhecida para nós. Talvez H. albicans pertença ao grupo monachica.

\section{Hippopsis prona Bates, 1866}

Hippopsis prona Bates, 1866: 41.

Hippopsis (Hippopsis) prona; Monné, 2005: 252 (cat.).

Hippopsis solangeae Carvalho, 1981b: 237, figs. 1-25. Syn. nov.

Hippopsis (Hippopsis) solangeae; Monné, 2005: 253 (cat.).

Gérard Tavakilian nos enviou fotografias do holótipo (MNHN), inclusive da face ventral do corpo. Constatamos, pelo orifício elíptico e profundo no urosternito I, que $H$. solangeae é sinônima de $H$. prona.

Chave para identificação das espécies de Hippopsis (não incluídas: $H$. lineolatus, $H$. lemniscata insularis e $H$. lemniscata boliviana).

1. Pronoto transversalmente rugoso (por exemplo, fig. 2)

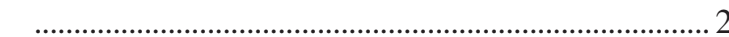

Pronoto sem rugas (por exemplo, fig. 3) ..................... 9

2(1). Protórax com quatro faixas longitudinais de 

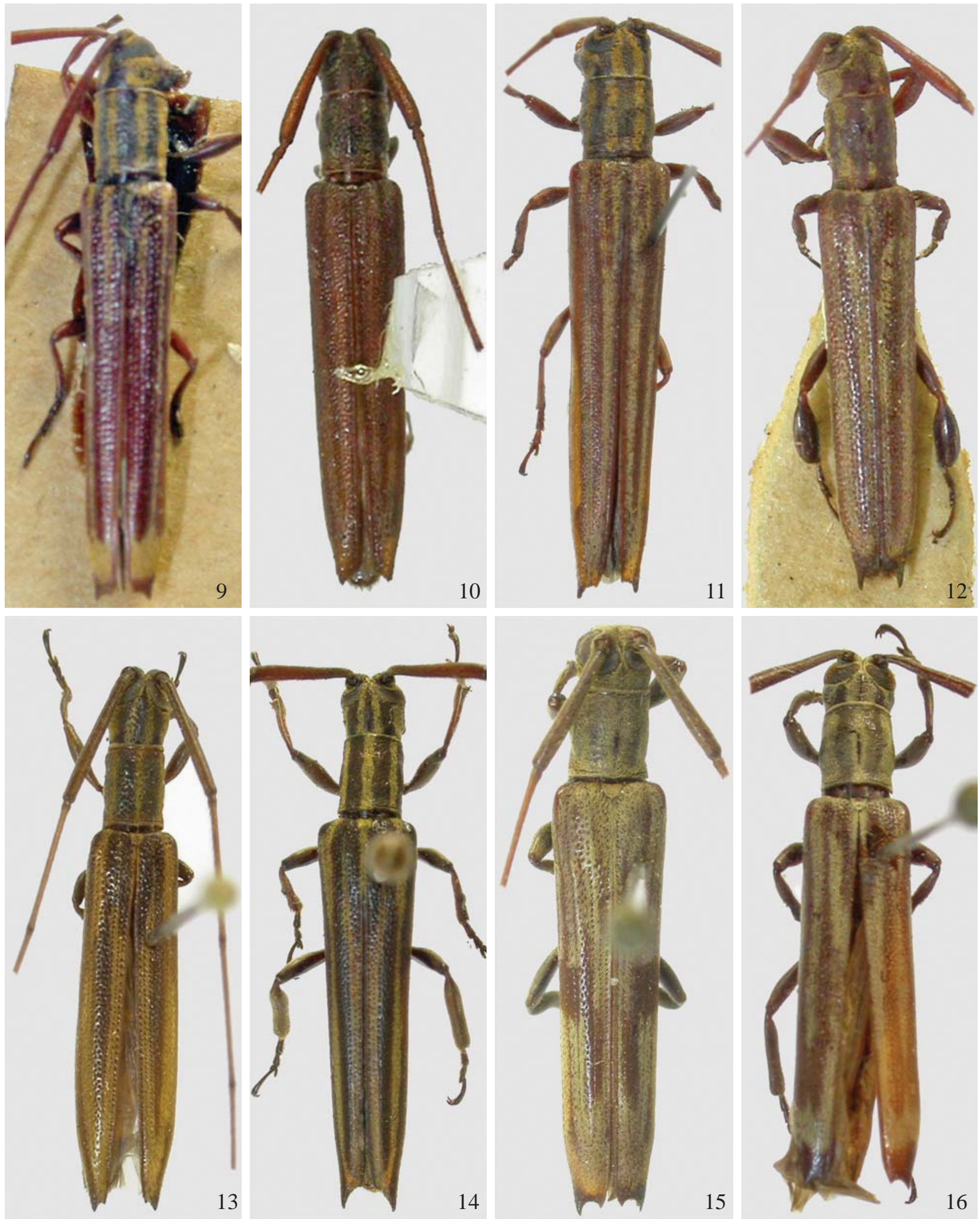

Figs. 9-16. 9, Hippopsis apicalis (Bates, 1866), comprimento 11,0 mm; 10, H. arriagadai Martins \& Galileo, 2003, holótipo macho, 7,4 mm; 11, H. brevicollis Martins \& Galileo, 2003, holótipo fêmea, 17,0 mm; 12, H. femoralis Breuning, 1940, macho, 8,7 mm; 13, H. ocularis Galileo \& Martins, 1995, holótipo fêmea, 9,5 mm; 14, H. quinquelineata Aurivillius, 1920, macho, 15,2 mm; 15, H. rabida Martins \& Galileo, 1988, parátipo macho, 12,1 mm; 16, H. renodis Martins \& Galileo, 1988, holótipo macho, 10,0 mm. 
pubescência amarelada 3

Protórax com seis ou sete faixas longitudinais de pubescência amarelada

3(2). Élitros com faixa de pubescência amarelada, longitudinal, que se inicia entre o escutelo e o úmero, contínua da base ao ápice; extremidade dos élitros cortada em curva com espinho externo ................. 4

Élitros com faixa de pubescência amarelada, longitudinal, que se inicia entre o escutelo e o úmero e termina no nível do terço posterior; extremidade dos élitros de per si acuminadas ou obliquamente truncadas .

5

4(3). Urosternito V sem modificações; élitros com faixa de pubescência amarelada que se inicia junto do escutelo curva-se para o lado da sutura no terço anterior e segue para o ápice fundida com a sutura (Fig. 1); ápices dos élitros com espinho externo e espículo sutural; centro dos urosternitos sem manchas longitudinais de pilosidade amarelada. Fig. 1. Venezuela ................. H. assimilis Breuning, 1940

Urosternito $\mathrm{V}$ com entalhe na borda apical ladeada por espículos (Figs. 49, 50); faixa de pubescência amarelada dos élitros que se inicia junto do escutelo não sofre curvaturas e segue distante da sutura até o sexto apical; centro dos urosternitos com faixa de pubescência amarelada. Fig. 36. Equador ...

H. iuasanga sp. nov.

5(3). Extremidades elitrais de per si acuminadas e projetadas em espinho longo; ponta dos metafêmures dos machos atingem a borda apical do urosternito I. Fig. 2. Peru, Equador, Brasil (Amazonas), Bolívia .. H. fractilinea Bates, 1866

Extremidades elitrais obliquamente truncadas e projetadas em espinho curto; ponta dos metafêmures dos machos atingem a borda apical do urosternito II. Fig. 5. Colômbia, Equador

H. quadrivittata Breuning, 1940

6(2). Extremidades dos élitros ocupadas por faixa escura, estreita. Fig. 3. Equador, Bolívia

H. nigroapicalis Martins \& Galileo, 2003

Extremidades dos élitros sem faixa escura 7

7(6). Protórax com sete faixas de pubescência amarelada, a do centro do pronoto bem indicada; extremidades elitrais cortadas em curva com espinho externo curto; (faixa de pubescência amarelada dos lados do metasterno separada da sutura metasternometepisternal). Fig. 6. Colômbia

H. septemlineata Breuning, 1940

Protórax com seis faixas de pubescência amarelada, a do centro do pronoto inexistente; extremidades elitrais com espinho externo longo ....
8(7). Faixa de pubescência amarelada dos lados do metasterno separada da sutura metasternometepisternal; pronoto com rugas mais profundas, evidentes, e sem pontos entremeados na faixa longitudinal do meio. Fig. 4. Brasil (Bahia a São Paulo) .............. H. pradieri Guérin-Méneville, 1844

Faixa de pubescência amarelada dos lados do metasterno sobre ou junto à sutura metasternometepisternal; pronoto com rugas mais rasas e com pontos entremeados na faixa longitudinal do meio. Fig. 34. Equador, Suriname, Brasil (Amazonas, Pará) H. macrophthalma Breuning, 1940

9(1). Extremidades elitrais cortadas em curva, com projeção ou espinho no lado externo ou com projeções em ambos os lados (por exemplo fig. 39) .................... 10

Extremidades elitrais de outro tipo, ou arredondadas ou projetadas em espinho .18

10(9). Protórax com faixas indistintas e pubescência quase uniforme, interrompida por vestígios de faixas escuras 11

Protórax com cinco ou seis faixas de pubescência amarelada ou branco-amarelada ............................ 12

11(10). Desenho elitral sem faixas longitudinais de pubescência amarelada e com uma área avermelhada, lateral, perto do meio; extremidades ocupadas por faixa escura; lobos oculares superiores (macho) separados entre si por distância equivalente a 1-2 omatídios. Figs. 15, 39. Brasil (São Paulo) .............. H. rabida Galileo \& Martins, 1988

Desenho elitral constituído por faixas de pubescência amarelada; extremidades elitrais sem faixa escura; lobos oculares superiores pouco mais distantes entre si do que a largura de um lobo. Fig. 10. Paraguai .... H. arriagadai Martins \& Galileo, 2003

12(10). Protórax com cinco faixas esbranquiçadas ou amareladas 13

Protórax com seis faixas amarelas .............................. 16

13(12). Extremidade dos élitros ocupadas por faixa escura; faixa central do pronoto larga, envolve área estreita, glabra e longitudinal; (face ventral do corpo com pubescência esbranquiçada uniforme) ................ 14

Extremidade dos élitros sem faixa escura; faixa do meio do pronoto estreita, não envolve área glabra ...... 15

14(13). Processo mesosternal estreito com largura igual a $1 / 3$ de uma mesocoxa; élitros com faixa brancoamarelada que se inicia entre o escutelo e o úmero, terminada no nível do terço posterior; metatíbias dos machos engrossadas e subcilíndricas. Fig. 16. Brasil (Rio de Janeiro) H. renodis Galileo \& Martins, 1988 

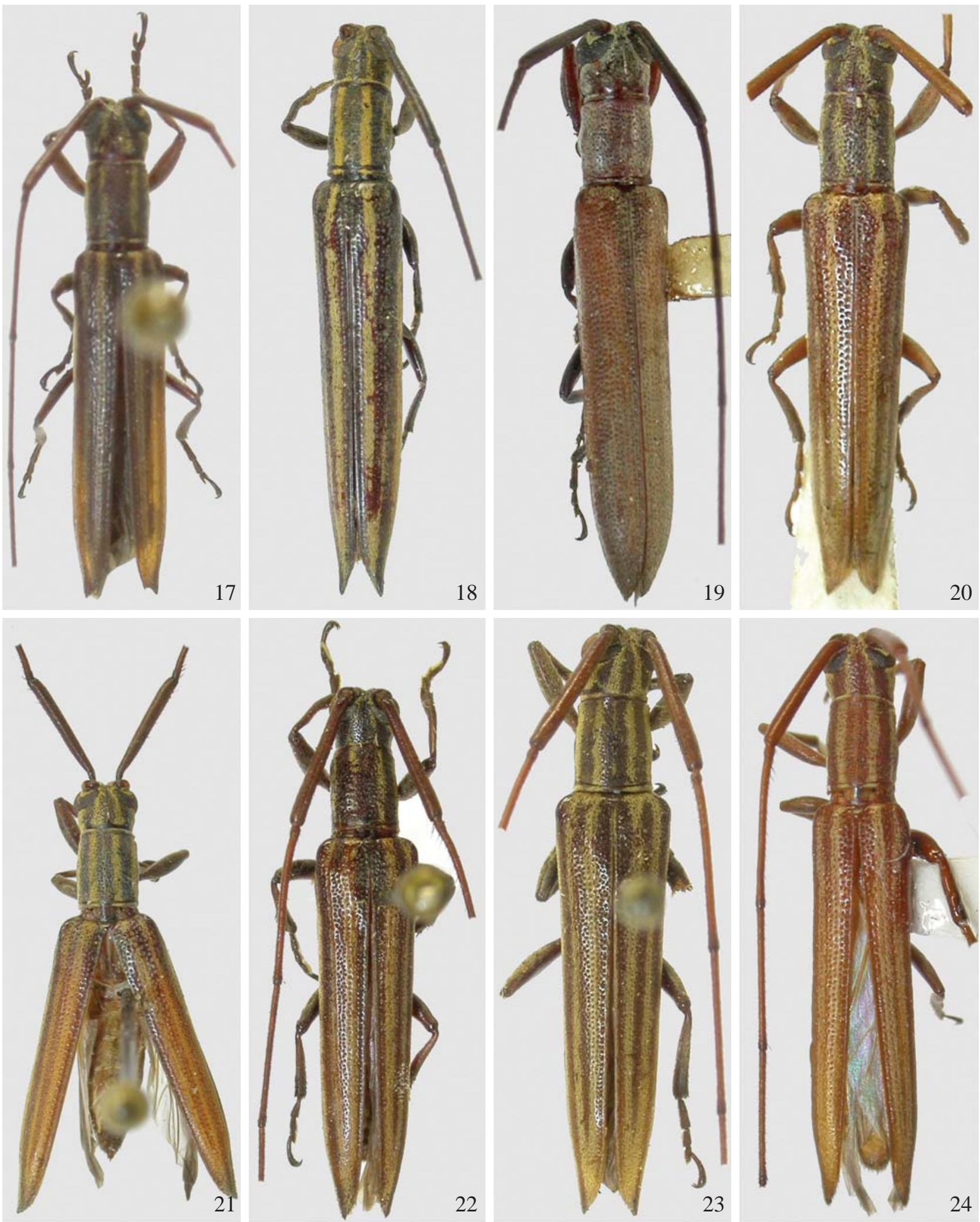

Figs. 17-24. 17, Hippopsis truncatella Bates, 1866, macho, 8,8 mm; 18, H. bivittata Martins \& Galileo, 2003, holótipo fêmea, 15,2 mm; 19, Hippopsis campaneri Martins \& Galileo, 1998, holótipo macho, comprimento 9,7 mm; 20, $H$. freyi Breuning, 1955, macho, 9,4 mm; $21, H$. meinerti Aurivillius, 1900, macho, 10,0 mm; 22, H. pertusa Galileo \& Martins, 1988, macho, 12,8 mm; 23, H. prona Bates, 1866, macho (parátipo de H. solangeae Carvalho, 1981); 24, H. tuberculata Galileo \& Martins, 1988, macho, 10,2 mm. 
Processo mesosternal com cerca de metade da largura de uma mesocoxa; élitros com faixas brancoamareladas contínuas; metatíbias dos machos fortemente globosas. Fig. 12. Brasil (Minas Gerais a Santa Catarina) ........ H. femoralis Breuning, 1940

15(13). Lobos oculares superiores contíguos; face inferior do escapo com franja de pêlos mais curtos que a sua largura basal. Fig. 13. Bolívia

H. ocularis Galileo \& Martins, 1995

Lobos oculares superiores não contíguos, separados por distância igual ao diâmetro de três omatídios; face inferior do escapo com pêlos tão longos quanto o diâmetro da sua base. Fig. 14. Brasil (Minas Gerais ao Rio Grande do Sul) .

H. quinquelineata Aurivillius, 1920

16(12). Extremidade dos élitros ocupada por faixa escura, precedida por faixa de pubescência amarelada. Fig. 9. Guiana Francesa, Equador, Brasil (Amazonas, Pará) ............................... H. apicalis (Bates, 1866)

Extremidade dos élitros sem faixa escura ................ 17

17(16). Maiores dimensões (comprimento, 17,0 mm); proporção comprimento do élitro/comprimento do protórax, 5,48; pronoto com duas gibosidades discretas à frente do meio. Fig. 11. Brasil (Minas Gerais) ....... H. brevicollis Martins \& Galileo, 2003

Menores dimensões (comprimento, 8,1-10,6 mm); proporção comprimento do élitro/comprimento do protórax, 4,20-4,45; pronoto sem gibosidades. Figs. 17, 37. Venezuela, Brasil (Amazonas, Pará, Rondônia, Mato Grosso, Mato Grosso do Sul, Goiás, Minas Gerais a Santa Catarina)

H. truncatella Bates, 1866

18(9). Extremidades elitrais arredondadas; (pubescência uniforme, sem faixas no pronoto e nos élitros). Brasil (Santa Catarina) .......... H. albicans Breuning, 1940 Extremidades elitrais acuminadas .... 19

19(18). Pêlos dos flagelômeros III e seguintes (Fig. 38) sinuosos e nitidamente mais longos que a largura dos artículos; élitros com pubescência amarelada sem faixas ou com faixas muito pouco contrastantes .. 20

Pêlos dos flagelômeros (Fig. 37) retos e mais curtos que a largura dos artículos; pubescência dos élitros organizadas em faixas longitudinais 21

20(19). Escapo com franja de pêlos no lado inferior; pêlos dos flagelômeros tão longos quanto o triplo da largura dos artículos. Figs. 7, 38. Brasil (Rio Grande do Sul), Uruguai, Argentina (Buenos Aires, Santa Fé) ...... H. monachica Berg, 1889

Escapo sem pêlos no lado inferior; pêlos dos flagelômeros tão longos quanto o dobro largura dos artículos. Fig. 8. Argentina (Córdoba)

H. pallida Carvalho, 1981

21(19). Cada élitro com apenas duas faixas longitudinais amareladas, uma junto à margem e uma perto da sutura; (protórax com quatro faixas de pubescência amarelada). Fig. 18. Peru

H. bivittata Martins \& Galileo, 2003

Cada élitro com três faixas de pubescência esbranquiçada ou amarelada, visíveis especialmente na metade apical dos élitros ou pubescência uniforme (não-organizada em faixas)

22(21). Região centro-anterior do metasterno com pequeno tubérculo; (urosternito I dos machos com orifício bem visível, fig. 47, por exemplo) ......................... 23

Região centro-anterior do metasterno sem tubérculo 24

23(22). Pubescência corporal uniforme, pronoto e élitros sem faixas longitudinais de pubescência; lobos oculares das fêmeas com seis fileiras de omatídios. Fig. 19. Brasil (Mato Grosso, Goiás) .....

H. campaneri Martins \& Galileo, 1998

Pubescência organizada em faixas no protórax e nos élitros; lobos oculares dos dois sexos com cinco fileiras de omatídios. Fig. 24. Brasil (Mato Grosso do Sul, Goiás, Minas Gerais a São Paulo), Bolívia, Argentina (Formosa)

H. tuberculata Galileo \& Martins, 1988

24(22). Protórax com sete faixas de pubescência brancoamarelada.... 25

Protórax com seis faixas de pubescência brancoamarelada. .. 26

25(24) Escutelo coberto por pubescência amarelada; élitros arredondados no ápice (Breuning, 1962: 7, 12). Brasil H. septemvittata Breuning, 1940

Escutelo coberto por pubescência serícea brancoamarelada e esparsa; ápice dos élitros oblíquo, com espinho curto no ângulo externo. Fig. 27. Estados Unidos, México, Honduras

H. l. lemniscata (Fabricius, 1801)

26(24). Tegumento avermelhado, faixas de pubescência esbranquiçada pouco aparentes nos élitros; (pequenas dimensões, comprimento, 9,2-10,5 mm). Fig. 33. Brasil (Pernambuco, Bahia)

H. fratercula Galileo \& Martins, 1988

Tegumento corporal castanho-avermelhado ou castanho; faixas de pubescência amareloesbranquiçada manifestas 27 

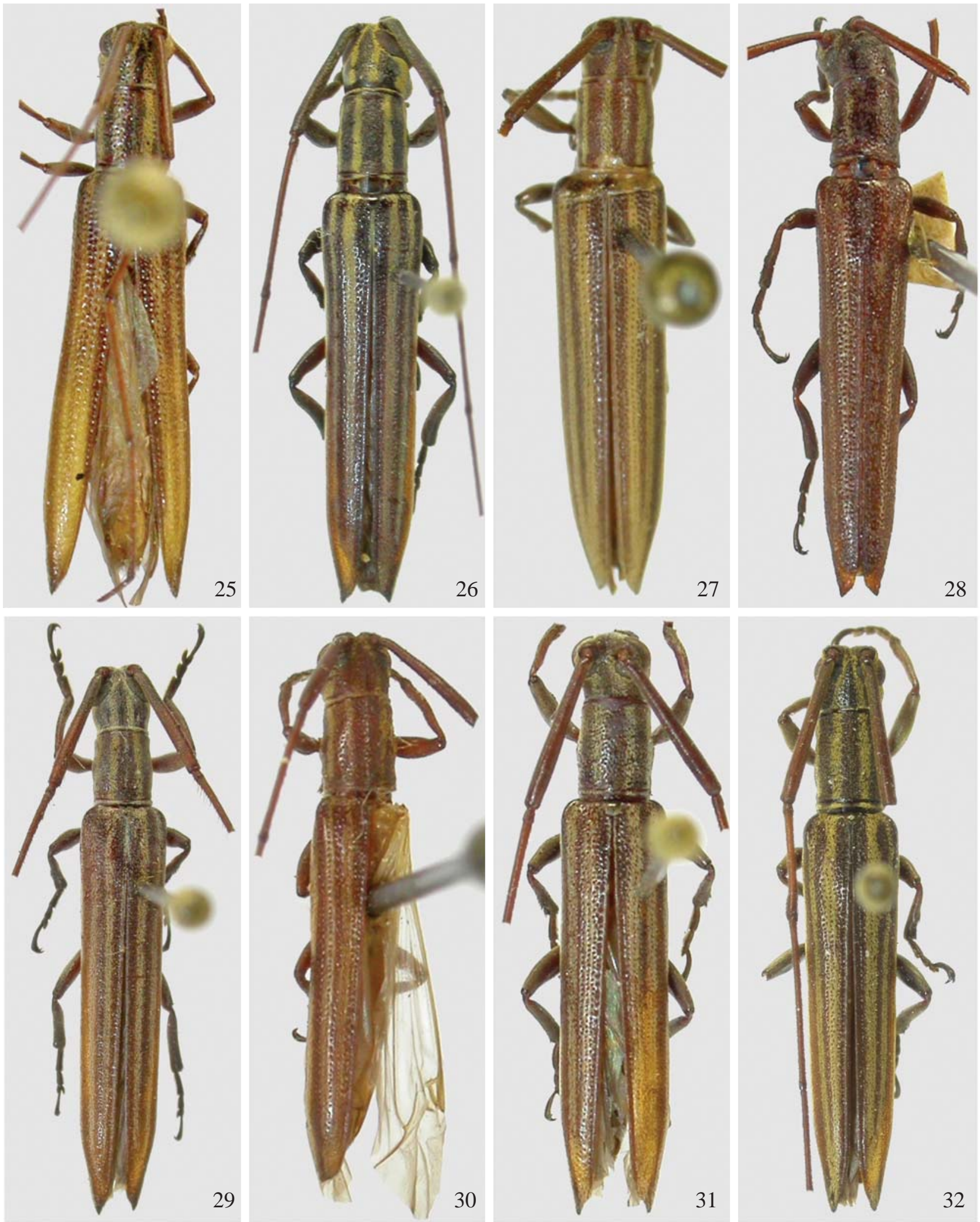

Figs. 25-32. 25, Hippopsis gilmouri Breuning, 1962, fêmea, comprimento 8,8 mm; 26, H. griseola Bates, 1866, 13,1 mm; 27, H. lemniscata lemniscata (Fabricius, 1801), fêmea, 11,3 mm; 28, H. densepunctata Breuning, 1940, macho, 9,2 mm; 29, H. tibialis Martins \& Galileo, 2003, holótipo macho, 13,2 mm; 30, H. minima Galileo \& Martins, 1988, macho, 8,1 mm; 31, H. mourai Martins \& Galileo, 1994, holótipo macho, $10,2 \mathrm{~mm} ; 32, \mathrm{H}$. pubiventris Galileo \& Martins, 1988, parátipo macho, 14,9 mm. 
27(26). Lobos oculares superiores com seis ou mais fileiras de omatídios ............................................................ 28

Lobos oculares superiores com cinco ou menos fileiras de omatídios .. 30

28(27). As três faixas de pubescência esbranquiçada dos élitros da mesma largura, isto é, a faixa que se inicia perto do úmero é da mesma largura que as duas outras. Fig. 29. Brasil (São Paulo) ...

H. tibialis Martins \& Galileo, 2003

A faixa de pubescência branco-amarelada dos élitros que se inicia próximo do úmero, mais estreita dos que as duas outras

29(28). Ápices dos élitros obliquamente truncados com espinho curto no lado externo; urosternito I dos machos sem modificações. Fig. 26. Brasil (Pará) ... H. griseola Bates, 1966

Ápices dos élitros de per si acuminados; urosternito I dos machos (Fig. 44) com área central de pilosidade diferenciada. Figs. 32, 44. Peru, Brasil (Acre a Santa Catarina) .... H. pubiventris Galileo \& Martins, 1988

30(27). Processo prosternal (Fig. 43) e processo mesosternal revestidos por pubescência amarelada, longa e dirigida para a parte posterior; macho: centro do metasterno com pêlos amarelados, longos, precedidos por área glabra, estreita; orifício do urosternito I como na figura 45. Fig. 22. Brasil (Ceará a Santa Catarina), Argentina (Tucumán, Catamarca) H. pertusa Galileo \& Martins, 1988

Pilosidade dos processos pro- e mesosternal normal; machos sem pêlos diferenciados no centro do metasterno 31

31(30). Face ventral densamente pontuada, inclusive os urosternitos 32

Face ventral com pontuação mais concentrada nos esternos torácicos; urosternitos praticamente sem pontos)

32(31). Faixas sutural e dorsal dos élitros separadas por linha acastanhada muito estreita apenas na metade anterior e fundidas a partir do meio. Fig. 35. Brasil (Bahia) Hippopsis araujoi $\mathrm{sp}$. nov.

Faixas sutural e dorsal dos élitros separadas por linha acastanhada até o quinto apical 33

33(32). Urosternito I dos machos com área central de pubescência diferenciada (como na fig. 44) ........ 34

Urosternito I sem pubescência diferenciada; (lobos oculares superiores mais afastados entre si do que a largura de um lobo). Fig. 27. Estados Unidos, México, Honduras

H. l. lemniscata (Fabricius, 1801)
34(33). Lobos oculares superiores tão distantes ente si quanto o dobro da largura de um lobo; metatíbias engrossadas e curvas. Fig. 28. Brasil (Bahia, Minas Gerais) ................ H. densepunctata Breuning, 1940

Lobos oculares superiores tão distantes entre si quanto a largura de um lobo; metatíbias engrossadas e retas. Fig. 31. Brasil (Tocantins) ....

H. mourai Martins \& Galileo, 1994

35(31). Metatarsômero I tão ou mais longo do que II+III .... 36 Metatarsômero I mais curto que II+III ..................... 37

36(35).Tegumento avermelhado; ápice dos élitros obliquamente truncados; (meio do urosternito I dos machos com área central diferenciada de pêlos). Fig. 30. Guiana, Brasil (Maranhão)

H. minima Galileo \& Martins, 1988

Tegumento acastanhado; ápice dos élitros de per si acuminados. Fig. 25. Argentina (Salta)

H. gilmouri Breunig, 1962

37(35). Macho. Metafêmures (Fig. 41) fusiformes, sem entalhe basal nem pêlos longos nessa região [Urosternito I (Fig. 40) com orifício elíptico, profundo, atinge o meio do segmento]. Fig. 23. Brasil (Amazonas, Pará, Mato Grosso, Goiás, Minas Gerais ao Paraná), Argentina (Chaco) ............. H. prona Bates, 1866

Macho. Metafêmures estreitados para o ápice, com entalhe basal e pêlos longos nessa região (Fig. 42)

38(37). Macho. Urosternito I (Fig. 48) com orifício losangular, com tubérculo no meio dos ramos laterais. Fêmea: Disco pronotal e base dos élitros com pontos justapostos, alguns anastomosados. Fig. 21. México a Costa Rica, Trinidad y Tobago, Venezuela, Guiana Francesa ......... H. meinerti Aurivillius, 1900

Macho. Urosternito I (Fig. 46) com orifício grande, quase atinge as bordas anterior e posterior. Fêmea: Disco pronotal e base dos élitros com pontos mais esparsos. Fig. 20. Colômbia, Trinidad y Tobago, Venezuela ............................ H. freyi Breuning, 1955

\section{Hippopsis iuasanga sp. nov.}

(Figs. 36, 49, 50)

Etimologia. Tupi, íu = espinho; asanga = curto. Alusivo aos espinhos no ápice do urosternito $\mathrm{V}$.

Fronte com pubescência amarelada no meio e junto aos lobos oculares inferiores. Tubérculos anteníferos próximos, projetados e agudos. Vértice com duas faixas de pubescência amarelada. Faixa de pubescência amarelada atrás dos olhos. Lobos oculares superiores com sete fileiras de omatídios.

Protórax com quatro faixas longitudinais de pubescência amarela: duas dorsais e duas no limite com o prosterno. 

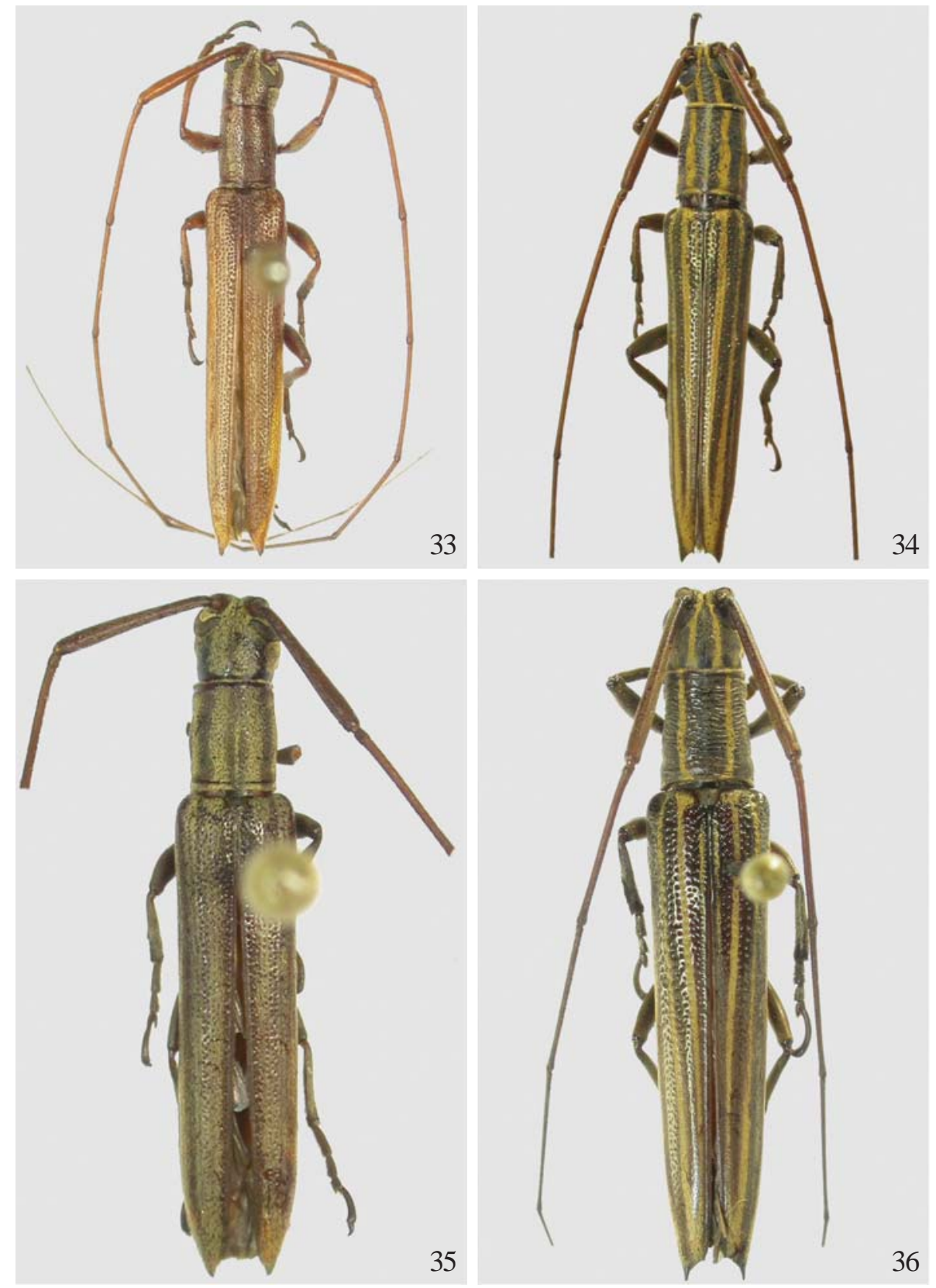

Figs. 33-36. Habitus: 33, Hippopsis fratercula Galileo \& Martins, 1988, parátipo macho, 11,2 mm; 34, H. macrophthalma Breuning, 1940, macho, $15,9 \mathrm{~mm} ; 35, H$. araujoi sp. nov., holótipo fêmea, $11,2 \mathrm{~mm} ; 36$, H. iuasanga sp. nov., holótipo fêmea, $22,5 \mathrm{~mm}$.

Pronoto com rugosidades transversais.

Cada élitro com três faixas estreitas de pubescência amarelada; metade anterior acentuadamente pontuada; ápice cortado em curva, com espinho externo.

Face ventral com pubescência amarelada: no lado superior do mesepisterno; no lado do metasterno; sobre a sutura metasterno-metepisternal; no meio e nos lados dos urosternitos. Ápice do urosternito V com recorte central semicircular (fêmea, fig. 50) ou em forma de "V" (macho, fig. 49), ladeado por dois espículos.

Dimensões em mm, parátipo macho/holótipo fêmea respectivamente. Comprimento total, 19,7/22,5; comprimento do protórax, 3,0/3,6; maior largura do protórax, 2,5/3,0; comprimento do élitro,14,7/16,3; largura umeral, 3,8/4,0.

Material-tipo. Holótipo fêmea, Equador, Pichincha: Tinalandia (15 km E Santo Domingo, 700 m), 23-26-XI.1981, H. F. Howden col. (FTHC); parátipo macho, Carchi: Chical $\left(00^{\circ} 56^{\prime} \mathrm{N} 78^{\circ} 11^{\prime} \mathrm{W}, 1250\right.$ m), 23.VII.1983, J. E. Rawlins col. (MZSP).

Discussão. H. iuasanga sp. nov. caracteriza-se pela forma do último urosternito com emarginação centro-apical com os ângulos espiniformes (figs. 49, 50). Até o momento nenhuma espécie do gênero Hippopsis apresenta modificações no último urosternito. 


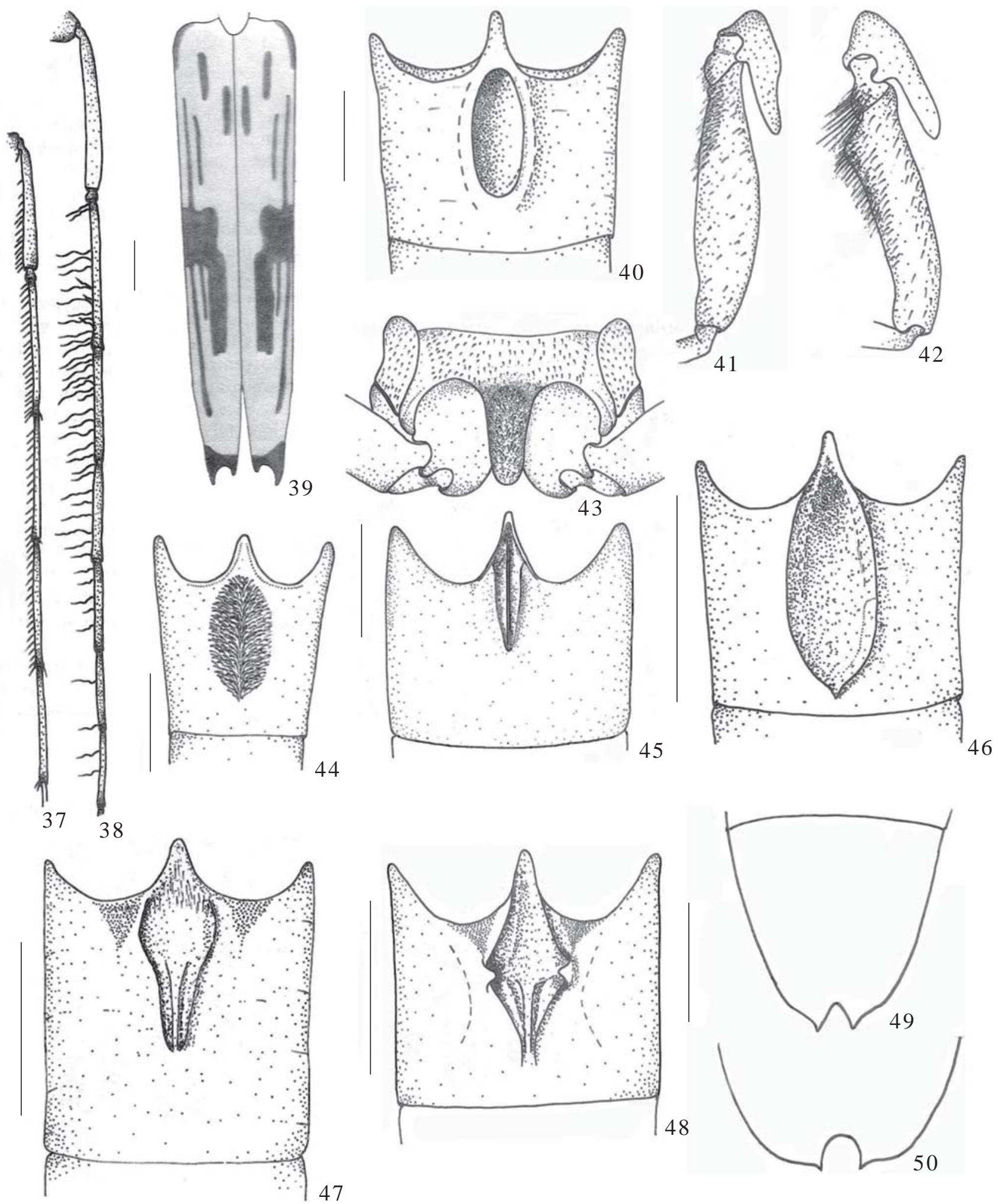

Figs. 37-50.Antenas: 37, Hippopsis truncatella; 38, H. monachica. Esquema do desenho elitral: 39, H. rabida. Vista ventral do urosternito I dos machos : 40, H. prona; 44, $\mathrm{H}$. pubiventris; 45, $\mathrm{H}$. pertusa; 46, $\mathrm{H}$. freyi; 47, $\mathrm{H}$. tuberculata; 48, $\mathrm{H}$. meinerti. Metafêmur do macho: 41, $H$. prona; 42, H. freyi. Prosterno: 43, H. pertusa. Vista ventral do urosternito V de $H$. iuasanga: 49, macho; 50, fêmea. Barra $=1 \mathrm{~mm}$. (Galileo \& Martins, 1988a, 1988b, 1988c). 


\section{Hippopsis araujoi sp. nov.}

(Fig. 35)

Etimologia. O nome específico é uma homenagem a Rafael Santos de Araujo que muito nos tem auxiliado na preparação de fotografias.

Fêmea. Tegumento acastanhado. Cabeça recoberta por pubescência densa, amarelada. Faixa subglabra atrás dos olhos. Tubérculos anteníferos discretos. Vértice inteiramente pubescente.

Protórax revestido por pubescência amarelada, mais concentrada em três faixas longitudinais de cada lado e uma linha no centro do pronoto.

Cada élitro com três faixas de pubescência amarelada: a sutural paralela e próxima à sutura; a dorsal discretamente separada da sutural na metade basal e fundida com ela na metade apical; a marginal paralela e pouco afastada da margem e interrompida atrás dos úmeros. Extremidades elitrais de per si acuminadas. Face ventral do corpo revestida por pubescência amarelada e densamente pontuada.

Dimensões mm, holótipo fêmea. Comprimento total, 11,2; comprimento do protórax, 1,9; maior largura do protórax, 1,4; comprimento do élitro, 8, 1; largura umeral, 1,9.

Material-tipo. Holótipo fêmea, Brasil, Bahia: Mucugé, 610.XII.1990, S. T. P. Amarante col. (MZSP).

Discussão. Distingue-se de $H$. mourai, pela pubescência corporal mais densa, pela faixa central de pubescência no pronoto e pelas faixas sutural e dorsal dos élitros fundidas no nível do meio. Em H. mourai a pubescência é mais esparsa, o pronoto não tem faixa central e as faixas elitrais sutural e dorsal fundem-se próximo do ápice.

Agradecimentos. A Rafael dos Santos Araujo e Luciano de Azevedo Moura, Museu de Ciências Naturais, Fundação Zoobotânica do Rio Grande do Sul pela execução e trato das ilustrações.

\section{REFERÊNCIAS}

Audinet-Serville, J. G. 1835. Nouvelle classification de la famille des longicornes (suite et fin). Annales de la Societé Entomologique de France 4: 197-228.

Aurivillius, C. 1900. Verzeichniss der von Dr. F. Meinert im Jahre 1891 in Venezuela gesammelten Cerambyciden. Öfversigt Svenska Vetenskaps-Academiens Förhandlingar 57: 409-421.

Aurivillius, C. 1919. Wissenchaftliche Ergebnisse der schwedischen entomologischen Reise des Herrn Dr. A. Roman in Amazons 19141915. Cerambyciden. Arkiv för Zoologi 12: 1-7.

Aurivillius, C. 1920. Neue oder wenig bekannte Coleoptera Longicornia. 17. Arkiv för Zoologi 13: 361-403.

Aurivillius, C. 1923. Coleopterorum Catalogus, pars 74, Cerambycidae: Lamiinae. Berlin, W. Junk, p. 323-704.

Bates, H. W. 1866. Contributions to an insect fauna of the Amazon Valley. Coleoptera: Longicornes. The Annals and Magazine of Natural History 17: 31-42.
Berg, C. 1889. Quadraginta Coleoptera nova Argentina. Anales de la Universidad de Buenos Aires 6: 105-157.

Breuning, S. 1940. Novae species Cerambycidarum VIII. Folia Zoologia et Hydrobiologica 10: 37-85.

Breuning, S. 1955. Neue Cerambyciden von der Insel Trinidad. Entomologischen Arbeiten aus der Museum G. Frey 6: 659661.

Breuning, S. 1962. Révision des Agapanthiini Muls. Américains (Col., Cerambycidae). Pesquisas 6: 1-48.

Carvalho, S. M. 1981a. Nova espécie de Hippopsis (Coleoptera, Cerambycidae, Laminae). Revista Brasileira de Entomologia 25: $53-54$.

Carvalho, S. M. 1981b. Hippopsis solangeae, sp. n. (Coleoptera, Cerambycidae) com notas sobre a morfologia do exoesqueleto. Revista Brasileira de Entomologia 25: 327-331.

Erichson, W. F. 1847. Conspectus insectorum coleopterorum quae in Republica Peruana observata sunt. Archiv für Naturgeschichte 13: $67-85$.

Galileo, M. H. M. \& U. R. Martins. 1988a. Notas sobre Agapanthiini (Coleoptera, Cerambycidae, Lamiinae). I. Hippopsis do grupo solangeae. Revista Brasileira de Entomologia 32: 179-185.

Galileo, M. H. M. \& U. R. Martins. 1988b. Notas sobre Agapanthiini (Coleoptera, Cerambycidae, Lamiinae). II. Hippopsis do grupo pubiventris. Revista Brasileira de Entomologia 32: 187-190.

Galileo, M. H. M. \& U. R. Martins. 1988c. Notas sobre Agapanthiini (Coleoptera, Cerambycidae, Lamiinae). III. Hippopsis do grupo lemniscata. Revista Brasileira de Entomologia 32: 191-195.

Galileo, M. H. M. \& U. R. Martins. 1988d. Notas sobre Agapanthiini (Coleoptera, Cerambycidae, Lamiinae). IV Hippopsis do grupo pradieri. Revista Brasileira de Entomologia 32: 196-198.

Galileo, M. H. M. \& U. R. Martins. 1988e. Notas sobre Agapanthiini (Coleoptera, Cerambycidae, Lamiinae). V Revisão do gênero Hippopsis Lepeletier \& Audinet-Serville, 1825. Revista Brasileira de Entomologia 32: 199-207.

Galileo, M. H. M. \& U. R. Martins. 1995. Novas espécies neotropicais da tribo Agapanthiini (Coleoptera, Cerambycidae). Iheringia, Zoologia, 78: 39-43.

Guérin-Méneville, F. E. 1844. Iconographie du règne animal de G. Cuvier... Paris, Baillière, Insectes 7: 5-76.

Kirch, T. F. 1875. Beitrage zur Kenntniss der Peruanischen Käferfauna auf Dr. Abendroth's basirt (Funftes Stuck). Deutsches Entomologischen Zeitschrift 19: 241-304.

Lepeletier, A. L. M. \& J. G. Audinet-Serville. 1825. In: Latreille, P. A. Encyclopédie méthodique, ou par ordre de matière, par un société de gens de lettres. Entomologie, 10: 1-344.

Martins, U. R. \& M. H. M. Galileo. 1994. Novas espécies e notas sobre Cerambycidae e Disteniidae (Coleoptera) do Estado de Tocantins, Brasil. Iheringia, Série Zoologia, 77: 77-82.

Martins, U. R. \& M. H. M. Galileo. 1998. Novas espécies neotropicais da tribo Agapanthiini (Coleoptera, Cerambycidae). Iheringia, Série Zoologia, 78: 39-43.

Martins, U. R. \& M. H. M. Galileo. 2003. Novas espécies e revalidação em Hippopsis Lepeletier \&Audinet-Serville, 1825 (Cerambycidae, Lamiinae, Agapanthiini). Revista Brasileira de Entomologia 47: $25-29$.

Monné, M. A. 2001. Catalogue of the Neotropical Cerambycidae (Coleoptera) with known host plant - Part III: Subfamily Lamiinae, tribes Acanthocinini to Apomecynini. Publicações Avulsas do Museu Nacional 92: 1-94.

Monné, M. A. 2005. Catalogue of the Cerambycidae (Coleoptera) of the Neotropical Region. Part II. Subfamily Lamiinae. Zootaxa 1023: $1-759$.

Monné, M. A. \& F. T. Hovore. 2005. Checklist of the Cerambycidae or longhorned woodboring beetles, of the Western Hemisphere. Rancho Dominguez, Bio Quip, 393 p.

Tavakilian, G. L. 1987. Nomenclatural changes, reinstatements, new combinations, and new synonymies among American Cerambycids (Coleoptera). Insecta Mundi 11: 129-139. 